\title{
SHRINAKGE FUNCTION AND ITS APPLICATIONS IN MATRIX APPROXIMATION
}

\author{
TOBY BOAS*, ARITRA DUTTA ${ }^{\dagger}, \mathrm{XIN}^{*}{ }^{\ddagger}, \mathrm{KATHRYN} \mathrm{P.} \mathrm{MERCIER}^{\S}$, AND ERIC \\ NIDERMAN $₫$
}

\begin{abstract}
The shrinkage function is widely used in matrix low-rank approximation, compressive sensing, and statistical estimation. In this article, an elementary derivation of the shrinkage function is given. In addition, applications of the shrinkage function are demonstrated in solving several well-known problems, together with a new result in matrix approximation.
\end{abstract}

Key words. Shrinkage function, singular value decomposition, low-rank approximation, sparse approximation.

AMS subject classifications. $65 \mathrm{~F} 15,65 \mathrm{~F} 30,65 \mathrm{~F} 35,65 \mathrm{~F} 50,65 \mathrm{~K} 10$

1. Introduction. Historically some important mathematical functions have been introduced for convenience. For example, the Heaviside step function $H(\cdot)$, a piecewise constant function given by:

$$
H(x)= \begin{cases}1, & x>0 \\ \frac{1}{2}, & x=0 \\ 0, & x<0\end{cases}
$$

and the Dirac delta function $\delta(\cdot)$ (more precisely, a distribution (see, e.g., [1])), a generalized function whose discrete analog is referred to as the Kronecker delta function:

$$
\delta_{i j}=\left\{\begin{array}{ll}
1, & i=j \\
0, & i \neq j
\end{array} .\right.
$$

This article concerns a newcomer, the shrinkage function $S_{\lambda}(\cdot)$, first introduced by Donoho and Johnstone in their landmark paper (2], see also [3]) on function estimation using wavelets in the early 1990's. Recently, the shrinkage function has been heavily used in the solutions of several optimization and approximation problems of matrices (see, e.g., 4, 5, 6, 7). We give an elementary treatment that is accessible to a vast group of researchers, as it only requires basic knowledge in calculus and linear algebra and show how naturally the shrinkage function can be used in solving more advanced problems.

2. A calculus problem. We start with a simple calculus problem. Let $\lambda>0$ and $a \in \mathbb{R}$ be given. Consider the following problem:

$$
\min _{x \in \mathbb{R}}\left[\lambda|x|+\frac{1}{2}(x-a)^{2}\right] .
$$

\footnotetext{
*Department of Mathematics, University of Florida, Gainesville, FL 32611-8105 (tboas@ufl.edu).

${ }^{\dagger}$ Department of Mathematics, University of Central Florida, 4000 Central Florida Blvd, Orlando, Florida 32816(d.aritra2010@knights.ucf.edu).

${ }^{\ddagger}$ Department of Mathematics, University of Central Florida, 4000 Central Florida Blvd, Orlando, Florida 32816 (xli@ucf.edu).

$\S^{\S}$ Department of Biology, University of Central Florida, 4000 Central Florida Blvd, Orlando, Florida 32816(katie.mercier@knights.ucf.edu).

IDepartment of Mathematics, University of Central Florida, 4000 Central Florida Blvd, Orlando, Florida 32816 (eaniederman@knights.ucf.edu).
} 
We adopt the notation $a=\arg \min _{x \in A} f(x)$ to mean that $a \in A$ is a solution of the minimization problem $\min _{x \in A} f(x)$ and define:

$$
S_{\lambda}(a):=\arg \min _{x \in \mathbb{R}}\left[\lambda|x|+\frac{1}{2}(x-a)^{2}\right] .
$$

Theorem 2.1. Let $\lambda>0$ be fixed. For each $a \in \mathbb{R}$, there is one and only one solution $S_{\lambda}(a)$, to the minimization problem (2.2). Furthermore,

$$
S_{\lambda}(a)= \begin{cases}a-\lambda, & a>\lambda \\ 0, & |a| \leq \lambda \\ a+\lambda, & a<-\lambda\end{cases}
$$

Remark. The function $S_{\lambda}(\cdot)$ defined above is called the shrinkage function (also referred to as soft shrinkage or soft threshold, [2, 3]). One may imagine that $S_{\lambda}(a)$ "shrinks" $a$ to zero when $|a| \leq \lambda$. A plot of $S_{\lambda}(\cdot)$ for $\lambda=1$ is given in Fig. 2.1.

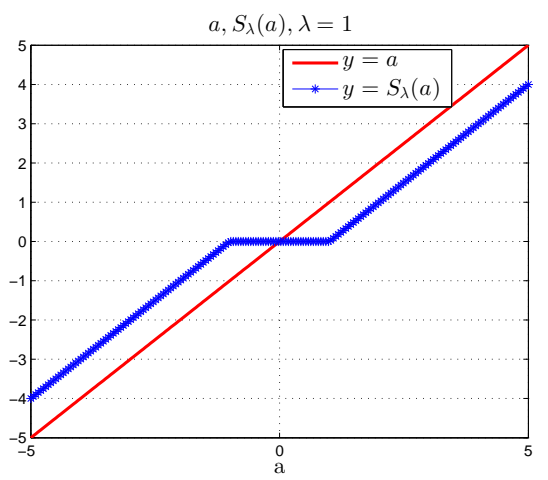

Fig. 2.1: A plot of $S_{\lambda}$ for $\lambda=1$.

Proof. Let $f(x)=\lambda|x|+\frac{1}{2}(x-a)^{2}$. Note that $f(x) \rightarrow \infty$ when $|x| \rightarrow \infty$ and $f$ is continuous on $\mathbb{R}$ and differentiable everywhere except a single point $x=0$. So, $f$ achieves its minimum value on $\mathbb{R}$ at one of its critical points. A plot of $f$ for different values of $a$ and $\lambda=1$ is given in Fig. 2.2. Let $x^{*}=\arg \min _{x \in \mathbb{R}} f(x)$.

We consider three cases.

Case 1: $x^{*}>0$. Since $f$ is differentiable at $x=x^{*}$ and achieves its minimum, we must have $f^{\prime}\left(x^{*}\right)=0$. Note that, for $x>0$, we have

$$
f^{\prime}(x)=\frac{d}{d x}\left[\lambda x+\frac{1}{2}(x-a)^{2}\right]=\lambda+(x-a) .
$$

So,

$$
\lambda+\left(x^{*}-a\right)=0,
$$

which implies

$$
x^{*}=a-\lambda .
$$




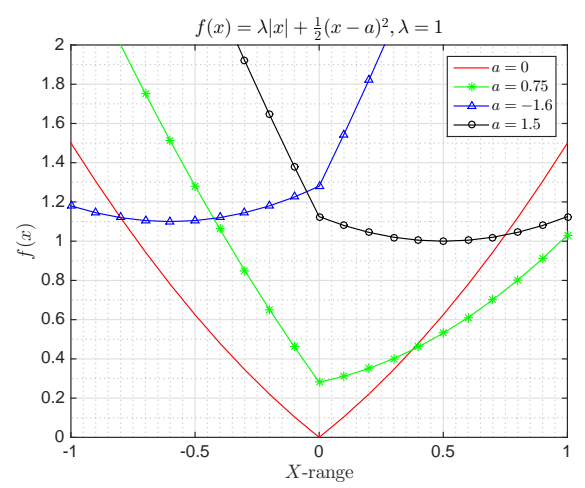

Fig. 2.2: Plots of $f(x)$ for different values of $a$ with $\lambda=1$.

To be consistent with $x^{*}>0$, it is necessary that $a-\lambda>0$ or, equivalently, $a>\lambda$.

Case 2: $x^{*}<0$. By proceeding similarly as in Case 1 above, we can arrive at

$$
x^{*}=a+\lambda \text { with } a<-\lambda .
$$

Case 3: $x^{*}=0$. Note that $f(x)$ is no longer differentiable at $x^{*}=0$ (so we could not use the condition $f^{\prime}\left(x^{*}\right)=0$ as before). But since $f$ has a minimum at $x^{*}=0$ and since $f$ is differentiable on each side of $x^{*}=0$, it is necessary that

$$
f^{\prime}(x)>0 \text { for } x>0 \text { and } f^{\prime}(x)<0 \text { for } x<0 .
$$

So,

$$
\lambda+x-a>0 \text { for } x>0 \text { and }-\lambda+x-a<0 \text { for } x<0 .
$$

Thus,

$$
\lambda-a>0 \text { and }-\lambda-a<0,
$$

or, equivalently,

$$
|a| \leq \lambda
$$

To summarize, we have

$$
x^{*}= \begin{cases}a-\lambda & \text { with } a>\lambda, \\ a+\lambda & \text { with } a<-\lambda, \\ 0 & \text { with }|a| \leq \lambda .\end{cases}
$$

Since one and only one of the three cases (1) $a>\lambda$, (2) $a<-\lambda$, and (3) $|a| \leq \lambda$ holds, we obtain the uniqueness. With the uniqueness, it is straightforward to verify that each of the three cases would imply the corresponding formula for $x^{*}$.

3. A sparse recovery problem. Recently, research in compressive sensing leads to the recognition of the fact that for many optimization problems, the $\ell_{1}$ norm of a vector is a good substitute for the count of the number of non-zero entries of the vector in many minimization problems. In this section, we solve some simple 
minimization problems using the count of non-zero entries or the $\ell_{1}$-norm. Given a vector $\mathbf{v} \in \mathbb{R}^{n}$, we want to solve

$$
\min _{\mathbf{u} \in \mathbb{R}^{n}}\left[\|\mathbf{u}\|_{\ell_{0}}+\frac{\beta}{2}\|\mathbf{u}-\mathbf{v}\|_{\ell_{2}}^{2}\right]
$$

where $\|\cdot\|_{\ell_{0}}$ denotes the number of non-zero entries of $\mathbf{u}$, such that, $\|\mathbf{u}\|_{\ell_{0}}=\#\left\{i \mid u_{i} \neq\right.$ $0\}$, the cardinality of $\mathbf{u},\|\cdot\|_{\ell_{2}}$ denotes the Euclidean norm in $\mathbb{R}^{n}$, and $\beta>0$ is a given balancing parameter. We can solve problem 3.1 component-wise (in each $u_{i}$ ) as follows. Notice that, given $\mathbf{u} \in \mathbb{R}^{n}$, each entry $u_{i}$ of $\mathbf{u}$ contributes 1 to $\|\mathbf{u}\|_{\ell_{0}}$ if $u_{i}$ is non-zero, and contributes 0 if $u_{i}$ is zero. Since we are minimizing $g(\mathbf{u}):=$ $\|\mathbf{u}\|_{\ell_{0}}+\frac{\beta}{2}\|\mathbf{u}-\mathbf{v}\|_{\ell_{2}}^{2}$, if $u_{i}$ is zero then the contribution to $g(\mathbf{u})$ depending on this $u_{i}$ is $\frac{\beta}{2} v_{i}^{2}$; otherwise, if $u_{i}$ is non-zero, then we should minimize $\frac{\beta}{2}\left(u_{i}-v_{i}\right)^{2}$ for $u_{i} \in \mathbb{R} \backslash\{0\}$, which forces that $u_{i}=v_{i}$ and contributes 1 to $g(\mathbf{u})$ as the minimum value. Therefore, the solution $\mathbf{u}$ to problem (3.1) is given component-wise by

$$
u_{i}=\left\{\begin{array}{lc}
0, & \text { if } \frac{\beta}{2}\left(v_{i}\right)^{2} \leq 1 \\
v_{i}, & \text { otherwise }
\end{array}\right.
$$

Next, we replace $\|\mathbf{u}\|_{\ell_{0}}$ by $\|\mathbf{u}\|_{\ell_{1}}$ in (3.1) and solve:

$$
\min _{\mathbf{u} \in \mathbb{R}^{n}}\left[\|\mathbf{u}\|_{\ell_{1}}+\frac{\beta}{2}\|\mathbf{u}-\mathbf{v}\|_{\ell_{2}}^{2}\right]
$$

where $\|\cdot\|_{\ell_{1}}$ denotes the $\ell_{1}$ norm in $\mathbb{R}^{n}$.

Using Theorem 2.1, we can solve (3.2) component-wise as follows.

TheOREM 3.1. [8] Let $\beta>0$ and $\mathbf{v} \in \mathbb{R}^{n}$ be given and let

$$
\mathbf{u}^{*}=\arg \min _{\mathbf{u} \in \mathbb{R}^{n}}\left[\|\mathbf{u}\|_{\ell_{1}}+\frac{\beta}{2}\|\mathbf{u}-\mathbf{v}\|_{\ell_{2}}^{2}\right]
$$

Then

$$
\mathbf{u}^{*}=S_{1 / \beta}(\mathbf{v}),
$$

where, $S_{1 / \beta}(\mathbf{v})$ denotes the vector whose entries are obtained by applying the shrinkage function $S_{1 / \beta}(\cdot)$ to the corresponding entries of $\mathbf{v}$.

Proof. If $u_{i}$ and $v_{i}$ denote the $i$ th entry of the vectors $\mathbf{u}$ and $\mathbf{v}$, respectively, $i=1,2, \ldots, n$, then we have,

$$
\begin{aligned}
\mathbf{u}^{*} & =\arg \min _{\mathbf{u} \in \mathbb{R}^{n}}\left[\|\mathbf{u}\|_{\ell_{1}}+\frac{\beta}{2}\|\mathbf{u}-\mathbf{v}\|_{\ell_{2}}^{2}\right] \\
& =\arg \min _{\mathbf{u} \in \mathbb{R}^{n}}\left[\sum_{i=1}^{n}\left(\frac{1}{\beta}\left|u_{i}\right|+\frac{1}{2}\left(u_{i}-v_{i}\right)^{2}\right)\right] .
\end{aligned}
$$

Since the $i$-th term in the summation depends only on $u_{i}$, the vector $\mathbf{u}^{*}$ must have components $u_{i}^{*}$ satisfying

$$
u_{i}^{*}=\arg \min _{u_{i}^{*} \in \mathbb{R}}\left[\frac{1}{\beta}\left|u_{i}\right|+\frac{1}{2}\left(u_{i}-v_{i}\right)^{2}\right],
$$

for $i=1,2, \ldots, n$. But by Theorem 2.1, the solution to each of these problems is given precisely by $S_{1 / \beta}\left(v_{i}\right)$. This yields the result. 
Remark. The previous proof still works if we replace the vectors by matrices and use the extension of the $\ell_{1}$ and $\ell_{2}$-norms to matrices by treating them as vectors. Thus by using the same argument we can easily show the following matrix version of the previous theorem.

Theorem 3.2. [8] Let $\beta>0$ and $\mathbf{V} \in \mathbb{R}^{m \times n}$ be given. Then

$$
S_{1 / \beta}(\mathbf{V})=\arg \min _{\mathbf{U} \in \mathbb{R}^{m \times n}}\left[\|\mathbf{U}\|_{\ell_{1}}+\frac{\beta}{2}\|\mathbf{U}-\mathbf{V}\|_{\ell_{2}}^{2}\right],
$$

where $S_{1 / \beta}(\mathbf{V})$ is defined component-wise.

Theorem 3.2 solves the problem of approximating a given matrix by a sparse matrix by using the shrinkage function.

4. Approximation by low rank matrices. The sparse approximation as given by Theorem 3.2 has many applications such as data compression and dimension reduction [9, 10. In these areas, one may also be interested in finding matrices of low rank (see, e.g., [5, 6, 7, 11). For example, given a matrix $\mathbf{A} \in \mathbb{R}^{m \times n}$, we want to solve the following approximation problem:

$$
\min _{\mathbf{X} \in \mathbb{R}^{m \times n}}\left[\operatorname{rank}(\mathbf{X})+\frac{\beta}{2}\|\mathbf{X}-\mathbf{A}\|_{F}^{2}\right],
$$

where $\|\cdot\|_{F}$ denotes the Frobenius norm of matrices (which turns out to be equivalent to the vector $l_{2}$ norm if we treat a matrix as a vector - see more discussion on the matrix norms in subsection 4.1).

This is a harder problem since $\operatorname{rank}(X)$ is not a convex function. A convex relaxation (see, e.g., [10]) of the problem is provided by replacing the term $\operatorname{rank}(\mathbf{X})$ by the nuclear norm of $\mathbf{X},\|\mathbf{X}\|_{*}$, (again, see subsection 4.1 for a discussion on the nuclear norm and its properties). The problem then becomes:

$$
\min _{\mathbf{X} \in \mathbb{R}^{m \times n}}\left[\|\mathbf{X}\|_{*}+\frac{\beta}{2}\|\mathbf{X}-\mathbf{A}\|_{F}^{2}\right] .
$$

This problem again yields an explicit solution (4, 12]), but in these literatures, the formula is derived by using advanced tools from convex analysis ("subdifferentials" to be more specific) [13. Here, we will show how we can obtain the solution by using simple ideas from the previous section.

4.1. Singular value decomposition and matrix norms. It will be beneficial to recall the various matrix norms. Many useful matrix norms can be defined in terms of the singular values of the matrices. We will deal with two of them: the nuclear norm $\|\cdot\|_{*}$ and the Frobenius norm $\|\cdot\|_{F}$.

Let $\mathbf{A} \in \mathbb{R}^{m \times n}$ and $\mathbf{A}=\mathbf{U} \tilde{\mathbf{A}} \mathbf{V}^{T}$ be a singular value decomposition (SVD) of $\mathbf{A}$ with $\mathbf{U} \in \mathbb{R}^{m \times m}$ and $\mathbf{V} \in \mathbb{R}^{n \times n}$ being two orthogonal matrices (that is, $\mathbf{U}^{-1}=\mathbf{U}^{T}$ and $\left.\mathbf{V}^{-1}=\mathbf{V}^{T}\right)$ and $\tilde{\mathbf{A}}=\operatorname{diag}\left(\sigma_{1}(\mathbf{A}), \sigma_{2}(\mathbf{A}), \ldots, \sigma_{\min \{m, n\}}(\mathbf{A})\right)$ being a $m \times n$ non-square diagonal matrix having $\sigma_{1}(\mathbf{A}) \geq \sigma_{2}(\mathbf{A}) \geq \cdots \geq \sigma_{\min \{m, n\}}(\mathbf{A}) \geq 0$ on its diagonal and 0's elsewhere. The $\sigma_{i}(\mathbf{A})$ 's are called the singular values of $\mathbf{A}$. It is known that every matrix in $\mathbb{R}^{m \times n}$ has a SVD and that SVD of a matrix is not necessarily unique [14. Then the nuclear norm of $\mathbf{A}$ is defined as

$$
\|\mathbf{A}\|_{*}=\sum_{i=1}^{\min \{m, n\}} \sigma_{i}(\mathbf{A}),
$$


and the Frobenius norm of $\mathbf{A}$ as

$$
\|\mathbf{A}\|_{F}=\left(\sum_{i=1}^{\min \{m, n\}}\left(\sigma_{i}(\mathbf{A})\right)^{2}\right)^{1 / 2}
$$

This norm turns out to be the same as the $\ell_{2}$ norm of $\mathbf{A}$, treated as a vector in $\mathbb{R}^{m n \times 1}$. This is because the nonzero singular values $\sigma_{i}(\mathbf{A})$ 's are exactly the square root of the nonzero eigenvalues of $\mathbf{A} \mathbf{A}^{T}$ or $\mathbf{A}^{T} \mathbf{A}$. So,

$$
\|\mathbf{A}\|_{l_{2}}^{2}=\sum_{i=1}^{m} \sum_{j=1}^{n}\left(a_{i j}\right)^{2}=\operatorname{trace}\left(\mathbf{A} \mathbf{A}^{T}\right)=\sum_{i=1}^{\min \{m, n\}}\left(\sigma_{i}(\mathbf{A})\right)^{2} .
$$

Here we have used trace $(\cdot)$ to denote the trace of a matrix (which is equal to the sum of all diagonal entries of the matrix).

We will need the following simple and well-known fact about the nuclear norms of a matrix and that of its diagonal [15]: Let $D(\mathbf{A})$ denote the diagonal matrix using the diagonal of $\mathbf{A}$ then:

$$
\|D(\mathbf{A})\|_{*} \leq\|\mathbf{A}\|_{*}
$$

This inequality can be verified by using a SVD of $\mathbf{A}=\mathbf{U} \tilde{\mathbf{A}} \mathbf{V}^{T}$ as follows. For completeness we will provide a simple proof of (4.2). Write $\mathbf{U}=\left(u_{i j}\right), \mathbf{V}=\left(v_{i j}\right)$, and $t=\min \{m, n\}$. Then

$$
\begin{gathered}
\|D(\mathbf{A})\|_{*}=\left\|D\left(\mathbf{U} \tilde{\mathbf{A}} \mathbf{V}^{T}\right)\right\|_{*}=\sum_{i=1}^{m}\left|\sum_{j=1}^{t} \sigma_{j}(\mathbf{A}) u_{i j} v_{i j}\right| \leq \sum_{j=1}^{t} \sigma_{j}(\mathbf{A}) \sum_{i=1}^{m}\left|u_{i j} v_{i j}\right| \\
\leq \sum_{j=1}^{t} \sigma_{j}(\mathbf{A}) \cdot\left(\sum_{i=1}^{m}\left|u_{i j}\right|^{2}\right)^{1 / 2}\left(\sum_{i=1}^{m}\left|v_{i j}\right|^{2}\right)^{1 / 2} \leq \sum_{j=1}^{t} \sigma_{j}(\mathbf{A})=\|\mathbf{A}\|_{*},
\end{gathered}
$$

where we have used the Cauchy-Schwarz inequality in obtaining the second inequality, and the orthogonality of $\mathbf{U}$ and $\mathbf{V}$ (so that $\sum_{i=1}^{m}\left|u_{i j}\right|^{2} \leq 1$ and $\sum_{i=1}^{m}\left|v_{i j}\right|^{2} \leq 1$ ) in the last inequality.

We will also use the fact that for any orthogonal matrices $\mathbf{L}$ and $\mathbf{R}, \mathbf{L A R}$ and A have the same singular values, and therefore their Frobenius norms and nuclear norms are same:

$$
\|\mathbf{L A R}\|_{F}=\|\mathbf{A}\|_{F} \text { and }\|\mathbf{L A R}\|_{*}=\|\mathbf{A}\|_{*} .
$$

This is known as the unitary invariance of the Frobenius norm and nuclear norm.

4.2. Solution to (4.1) via problem (2.2). We are ready to show how problem (4.1) is problem (2.2) in disguise. Let $\mathbf{U} \tilde{\mathbf{A}} \mathbf{V}^{T}$ be a SVD of $\mathbf{A}$. Given $\beta>0$, using the unitary invariance of the Frobenius norm and the nuclear norm, we have

$$
\begin{aligned}
\min _{\mathbf{X}}\left[\|\mathbf{X}\|_{*}+\frac{\beta}{2}\|\mathbf{X}-\mathbf{A}\|_{F}^{2}\right] & =\min _{\mathbf{X}}\left[\|\mathbf{X}\|_{*}+\frac{\beta}{2}\left\|\mathbf{X}-\mathbf{U} \tilde{\mathbf{A}} \mathbf{V}^{T}\right\|_{F}^{2}\right] \\
& =\min _{\mathbf{X}}\left[\left\|\mathbf{U}^{T} \mathbf{X V}\right\|_{*}+\frac{\beta}{2}\left\|\mathbf{U}^{T} \mathbf{X} \mathbf{V}-\tilde{\mathbf{A}}\right\|_{F}^{2}\right] .
\end{aligned}
$$


It can be seen from the last expression that the minimum occurs when $\tilde{\mathbf{X}}:=\mathbf{U}^{T} \mathbf{X V}$ is diagonal since both terms in that expression get no larger when $\tilde{\mathbf{X}}$ is replaced by its diagonal matrix (with the help of (4.2)). So, the matrix $\mathbf{E}=\left(e_{i j}\right):=\tilde{\mathbf{X}}-\tilde{\mathbf{A}}$ is a diagonal matrix. Thus,

$$
\mathbf{X}=\mathbf{U} \tilde{\mathbf{X}} \mathbf{V}^{T} \text {, with } \tilde{\mathbf{X}}=\tilde{\mathbf{A}}+\mathbf{E},
$$

which yields a SVD of $\mathbf{X}$ (using the same matrices $\mathbf{U}$ and $\mathbf{V}$ as in a SVD of $\mathbf{A}$ !). Then,

$$
\begin{aligned}
\min _{\mathbf{X}}\left[\|\mathbf{X}\|_{*}+\frac{\beta}{2}\|\mathbf{X}-\mathbf{A}\|_{F}^{2}\right] & =\min _{\tilde{\mathbf{X}} \in \operatorname{diag}}\left[\|\tilde{\mathbf{X}}\|_{*}+\frac{\beta}{2}\|\tilde{\mathbf{X}}-\tilde{\mathbf{A}}\|_{F}^{2}\right] \\
& =\min _{\tilde{\mathbf{X}} \in \operatorname{diag}}\left[\sum_{i}\left|\tilde{x}_{i i}\right|+\frac{\beta}{2} \sum_{i}\left(\tilde{x}_{i i}-\sigma_{i}(\mathbf{A})\right)^{2}\right],
\end{aligned}
$$

where "diag" is the set of diagonal matrices in $\mathbb{R}^{m \times n}$. Note that (4.3) is an optimization problem like (2.2) (for vectors $\left(\tilde{x}_{11}, \tilde{x}_{22}, \cdots\right)^{T}$ as $\tilde{\mathbf{X}}$ varies) whose solution is given by

$$
\tilde{x}_{i i}=S_{1 / \beta}\left(\sigma_{i}(\mathbf{A})\right), i=1,2, \cdots .
$$

To summarize, we have proven the following.

Theorem 4.1. 4] Suppose that $\mathbf{A} \in \mathbb{R}^{m \times n}$ and $\beta>0$ are given. Then the solution to the minimization problem (4.1) is given by $\hat{\mathbf{X}}=\mathbf{U} \tilde{\mathbf{X}} \mathbf{V}^{T}$ where the diagonal matrix $\tilde{\mathbf{X}}$ has diagonal entries

$$
\tilde{x}_{i i}=S_{1 / \beta}\left(\sigma_{i}(\mathbf{A})\right), i=1,2, \ldots, \min \{m, n\},
$$

where $\mathbf{U} \tilde{\mathbf{A}} \mathbf{V}^{T}$ is a $S V D$ of $\mathbf{A}$.

Remark. 1. A recent proof of this theorem is given by Cai, Candes, and Shen in [4] where they give an advanced verification of the result. Our proof given above has the advantage that it is elementary and allows the reader to "discover" the result.

2. There are many earlier discoveries of related results ([14, 16]) where $\operatorname{rank}(\mathbf{X})$ is used instead of the nuclear norm $\|\mathbf{X}\|_{*}$. We will examine one such variant in the next section.

3. A closely related (but harder) problem is compressive sensing ([17, 18]). In a more general set up where only a subset of the entries of the data matrix is observable, for example, matrix completion problem under low-rank penalties [4, 12, 18]:

$$
\min _{\mathbf{X}} \operatorname{rank}(\mathbf{X}) \quad \text { subjcet to } A_{i j}=X_{i j},(i, j) \in \Omega,
$$

where $\Omega \subseteq\{(i, j): 1 \leq i \leq m, 1 \leq j \leq n\}$, is indeed NP-hard [19]. Note that, the matrix completion problem is a special case of the affinely constrained matrix rank minimization problem [12]:

$$
\min _{\mathbf{X}} \operatorname{rank}(\mathbf{X}) \quad \text { subjcet to } A(\mathbf{X})=\mathbf{b},
$$

where $\mathbf{X} \in \mathbb{R}^{m \times n}$ be the decision variable and $\mathbf{A}: \mathbb{R}^{m \times n} \rightarrow \mathbb{R}^{p}$ be a linear map. One common idea used in such a situation is to consider a convex relaxation of the above problem by replacing the rank by its convex surrogate, the nuclear norm. Readers are strongly recommended to the recently survey by Bryan and Leise (9]). 
5. A variation. More problems can be solved by applying similar ideas. For example, let us consider a variant of a well-known result of Schmidt (see, e.g., [16, Section 5]), replacing the rank by the nuclear norm: For a fixed positive number $\tau$, consider

$$
\min _{\mathbf{X} \in \mathbb{R}^{m \times n}}\|\mathbf{X}-\mathbf{A}\|_{F} \text { subject to }\|\mathbf{X}\|_{*} \leq \tau .
$$

Using similar methods as in Section 3, this problem can be transformed (see the derivation below) into the following:

$$
\min _{\mathbf{u} \in \mathbb{R}^{\min \{m, n\}}}\|\mathbf{u}-\mathbf{v}\|_{\ell_{2}} \text { subject to }\|\mathbf{u}\|_{\ell_{1}} \leq \tau .
$$

The LASSO (Least absolute shrinkage and selection operator) is a vastly used regression technique in data mining and statistics [3, 20, 21. It follows a simple model for variable selection and regularization. Let the predictor variables, $\mathbf{X} \in \mathbb{R}^{N \times p}$ and the responses, $y_{i}, i=1,2, \ldots, N$ are given. Assuming that $\sum_{i} x_{i j} / N=0$ and $\sum_{i} x_{i j}^{2} / N=1$, the LASSO estimate is given by:

$$
\hat{\beta}=\arg \min _{\beta}\left\|\mathbf{y}-\beta_{\mathbf{0}}-\mathbf{X} \beta\right\|_{\ell_{2}}^{2} \text {, subject to }\|\beta\|_{\ell_{1}} \leq \tau,
$$

where $\beta_{0}$ is the mean of the response vector $\mathbf{y}$. Note that, (5.2) shares some similarity with (5.3) [3, 20, 21]. As in [3, one can form a Lagrangian of (5.2) and solve:

$$
\mathbf{u}^{*}=\arg \min _{\mathbf{u} \in \mathbb{R}^{\min \{m, n\}}}\left\{\frac{1}{2}\|\mathbf{u}-\mathbf{v}\|_{\ell_{2}}^{2}+\lambda\|\mathbf{u}\|_{\ell_{1}}\right\}, \quad \text { with }\left\|S_{\lambda}(\mathbf{v})\right\|_{\ell_{1}}=\tau,
$$

which has a solution $\mathbf{u}^{*}=S_{\lambda}(\mathbf{v})$ according to Theorem 3.1. (The reason for us to use $\lambda$ instead of $\beta$ in (5.2) is nonessential: it is only for indicating the similarity with LASSO formulation.) We now sketch the derivation of converting (5.1) to (5.2): As before, let $\mathbf{A}=\mathbf{U} \tilde{\mathbf{A}} \mathbf{V}^{T}$ be a SVD of $\mathbf{A}$. Then,

$$
\min _{\mathbf{X} \in \mathbb{R}^{m \times n}}\|\mathbf{X}-\mathbf{A}\|_{F}=\min _{\mathbf{X} \in \mathbb{R}^{m \times n}}\left\|\mathbf{U}^{\mathbf{T}} \mathbf{X V}-\tilde{\mathbf{A}}\right\|_{F} .
$$

Note that $\|\mathbf{X}\|_{*}=\left\|\mathbf{U}^{T} \mathbf{X V}\right\|_{*}$, so (5.1) can be written as

$$
\min _{\mathbf{X} \in \mathbb{R}^{m \times n}}\|\mathbf{X}-\tilde{\mathbf{A}}\|_{F} \text { subject to }\|\mathbf{X}\|_{*} \leq \tau,
$$

which, by using (4.2), can be further transformed to

$$
\min _{\mathbf{X} \in \mathbb{R}^{m \times n}}\|\mathbf{X}-\tilde{\mathbf{A}}\|_{F} \quad \text { subject to } \mathbf{X} \text { being diagonal and }\|\mathbf{X}\|_{*} \leq \tau \text {. }
$$

Next, if we let $\mathbf{u}$ and $\mathbf{v}$ be two vectors in $\mathbb{R}^{\min \{m, n\}}$ consisting of the diagonal elements of $\mathbf{X}$ and $\tilde{\mathbf{A}}$, respectively, then (5.5) is equivalent to (5.2). Since $S_{\lambda_{1}}(\mathbf{v})$ solves (5.4) we have,

$$
\frac{1}{2}\left\|S_{\lambda_{1}}(\mathbf{v})-\mathbf{v}\right\|_{2}^{2}+\lambda_{1} \tau \leq \frac{1}{2}\|\mathbf{u}-\mathbf{v}\|_{\ell_{2}}^{2}+\lambda_{1}\|\mathbf{u}\|_{\ell_{1}},
$$

for all $\mathbf{u} \in \mathbb{R}^{\min \{m, n\}}$. Which implies, for all $\mathbf{u} \in \mathbb{R}^{\min \{m, n\}}$,

$$
\frac{1}{2}\left\|S_{\lambda_{1}}(\mathbf{v})-\mathbf{v}\right\|_{2}^{2} \leq \frac{1}{2}\|\mathbf{u}-\mathbf{v}\|_{\ell_{2}}^{2}+\lambda_{1}\left(\|\mathbf{u}\|_{\ell_{1}}-\tau\right) .
$$


Therefore,

$$
\frac{1}{2}\left\|S_{\lambda_{1}}(\mathbf{v})-\mathbf{v}\right\|_{2}^{2} \leq\left\{\frac{1}{2}\|\mathbf{u}-\mathbf{v}\|_{\ell_{2}}^{2}\right\},
$$

for all $\mathbf{u} \in \mathbb{R}^{\min \{m, n\}}$, such that $\|\mathbf{u}\|_{\ell_{1}} \leq \tau$. Hence $\mathbf{u}^{*}=S_{\lambda}(\mathbf{v})$ solves (5.2). Thus we have established the following result.

THEOREM 5.1. [11] With the notations above, the solution to problem [5.1) is given by

$$
\hat{\mathbf{X}}=\mathbf{U} S_{\lambda}(\tilde{\mathbf{A}}) \mathbf{V}^{T},
$$

for some $\lambda$ such that $\left\|S_{\lambda}(\tilde{\mathbf{A}})\right\|_{\ell_{1}}=\tau$.

Acknowledgment. This work is partially supported by a CSUMS program of the National Science Foundation DMS-0803059. Toby Boas, Katie Mercier, and Eric Niederman contributed to this work as undergraduate students.

\section{REFERENCES}

[1] Bracewell, R., The Impulse Symbol, The Fourier Transform and Its Applications (3rd ed., 2000), Ch. 5, McGraw-Hill, pp. 74-104.

[2] Donoho, D. L. And Johnstone, I. M., Ideal spatial adaptation by wavelet shrinkage, Biometrika, 81 (1994),pp. 425-455.

[3] Tibshirani, R., Regression shrinkage and selection via the LASSO, Journal of the Royal statistical society, series B, 58 (1996), pp. 267-288.

[4] Cai, J., Candes, E. J. And Shen, Z., A singular value thresholding algorithm for matrix completion, SIAM Journal on Optimization, 20 (2010), pp. 1956-1982.

[5] Lin, Z., Chen, M. And Ma, Y., The augmented Lagrange multiplier method for exact recovery of corrupted low-rank matrices, arXiv:1009.5055v2, March 9, 2011.

[6] TaO, M. And YuAN, X., Recovering low-rank and sparse components of matrices from incomplete and noisy observations, SIAM Journal on Optimization, 21 (2011), pp. 57-81.

[7] YUAN, X. AND YANG, J., Sparse and low-rank matrix decomposition via alternating direction methods, Technical report (available from http://www.optimization-online.org/DBFILE/2009/11/2447.pdf), Dept. of Mathematics, Hong Kong Baptist University, 2009.

[8] Yin, W., Hale, E. And Zhang, Y., Fixed-point continuation for $l_{1}$-minimization: methodology and convergence, SIAM Journal on Optimization, 19 (2008), pp. 1107-1130.

[9] Bryan, K. AND Leise, T., Making do with less: an introduction to compressed sensing, SIAM Review, 55 (2013), pp. 547-566.

[10] Fazel, M., Matrix Rank Minimization with Applications, Ph.D. dissertation, Department of Electrical Engineering, Stanford University, 2002.

[11] Dutta, A., Weighted low-rank approximation of matrices: some analytical and numerical aspects, Ph.D. dissertation, Department of Mathematics, University of Central Florida, 2016.

[12] Ma, S. Q., Goldfarb, D. And Chen, L. F., Fixed point and Bergman iterative methods for matrix rank minimization, Mathematical Programming Series A, 128-1 (2011), pp. 321-353.

[13] Watson, G.A., Characterization of the subdifferential of some matrix norms, Linear Algebra and its Applications, 170 (1992), pp. 33-45.

[14] Strang, G. W., Introduction to Linear Algebra, 3rd ed., Wellesley-Cambridge Press, 1998.

[15] Stewart, G.W. And Sun, J.,Matrix perturbation theory, Academic Press, Boston, 1990.

[16] Stewart, G. W., On the early history of the singular value decomposition, SIAM Review, 35 (1993), pp. 551-566.

[17] Romberg, J. AND TAO, T., Robust uncertainty principles: Exact signal reconstruction from highly incomplete frequency information, IEEE Transactions on Information Theory, 52 (2006), pp. 489-509.

[18] Candes, E. J. And TaO, T., The power of convex relaxation: Near-optimal matrix completion, IEEE Transactions on Information Theory, 56 (2010), pp. 2053-2080. 
[19] Chistov, A. L. And Grigor'ev, D. Yu., Complexity of quantifier elimination in the theory of algebraically closed fields, Mathematical Foundations of Computer Science's Lecture Notes in Computer Science, 176 (1984), pp. 17-31.

[20] Tibshirani, R. J. And Taylor, J., The solution path of the generalized LASSO, The Annals of Statistics, 39-3 (2011), pp. 1335-1371.

[21] Osborne, M. R., Presnell, B. and Turlach, B. A., On the LASSO and its dual, Journal of Computational and Graphical Stat, 9 (1999), pp. 319-337. 